\title{
Two- and four-relay selection schemes for application in interference limited legacy networks
}

\author{
Gaojie Chen ${ }^{*}$, Ousama Alnatouh ${ }^{*}$ and Jonathon Chambers
}

\begin{abstract}
The limiting effect of multi-user interference from an adjacent cell upon the relays used for cooperative transmission is considered in the context of legacy networks which adopt $\max (\min (\cdot, \cdot))$ type relay selection policies. We extend previous work which considered single relay selection to the selection of two or four such relays, as is required in distributed space-time coding. We obtain new analytical expressions for outage probability over Rayleigh frequency flat fading channels for two signal-to-noise ratio regimes. We confirm by simulation that such a relay selection scheme has robustness to relay selection feedback error and outperforms a single relay selection scheme.
\end{abstract}

\section{Introduction}

Cooperative relaying can be considered as an effective method to combat fading by exploiting spatial diversity [1], and as a way for two users with no or weak direct connection to attain a robust link. One or more relay nodes are generally used in such relaying to forward signals transmitted from the source node to the destination node. In a cooperative communication system, there are two main cooperative methods: decode-and-forward (DF) (regenerative relaying protocol) and amplify-and-forward (AF) (transparent relaying protocol) methods [2]. In the DF method, relay nodes decode the source information and then re-encode and re-transmit it to the destination. In the AF method, relay nodes only amplify and retransmit their received signals, including noise, to the destination. Therefore, compared with DF, AF-type schemes have the advantage of simple implementation and low complexity in practical scenarios. In addition to complexity benefits, it has been shown in [3] that an AF scheme asymptotically, in terms of appropriate power control, approaches a DF one with respect to diversity.

AF has extensively been studied in the literature, but generally in the context of ideal configurations without interference during the cooperation process $[4,5]$. However, more practical systems have been studied in

*Correspondence: g.chen@lboro.co.uk; o.alnatouh@lboro.co.uk School of Electronic, Electrical and Systems Engineering, Loughborough University, Loughborough, UK
[6-8], which consider the presence of interference effects. The effect of cochannel interference on the performance of multihop wireless networks with AF relaying is analyzed in [6]. The performance of a two-hop channel state information-assisted AF system, with co-channel interference at the relay, is analyzed in [8].

In a cooperative relay network, moveover, when many relays can help the source to transmit to the destination, sometimes some relays provide a poor channel quality which can affect the end-to-end transmission quality [9]. Therefore, the use of a relay selection scheme is attracting considerable attention to overcome this problem and preserve the potential diversity gains [10-12], while mitigating the problem in synchronizing a large number of cooperative nodes.

In [10], exact outage and diversity performance expressions for a single relay selection scheme are provided for a wide range of signal-to-noise ratio (SNR) regimes in the context of an AF transmission protocol. The work in [11] relies on using instantaneous end-to-end wireless channel conditions to obtain the best single relay for cooperative diversity. This study was extended in [12] to obtain outage-optimal opportunistic relaying in the context of selecting a single relay from a set of $N$ available relays. They show that cooperative diversity gain is achieved even when certain relays remain inactive. However, these relay selection criteria lack the flexibility to deal with the presence or absence of interference effects.

\section{Springer}

(c) 2012 Chen et al:; licensee Springer. This is an Open Access article distributed under the terms of the Creative Commons Attribution License (http://creativecommons.org/licenses/by/2.0), which permits unrestricted use, distribution, and reproduction in any medium, provided the original work is properly cited. 
In order to improve the practicality, in [13] the effects of multi-user interference are considered for relay nodes and a single relay selection scheme is used to overcome the effects of the interference, in the context of legacy networks. However, using a single best relay is not always sufficient to satisfy the required outage probability at a destination node. Moreover, these works have not considered feedback error for relay selection, which means sometimes the best relay cannot be chosen because the wrong enable feedback information is received from the destination node. We highlight that this is different from recent work which has considered the effect of only delay in the feedback path [14].

Therefore, in this article, in order to overcome these shortcomings, first, the basic AF protocol [15] is considered when external out-of-cell structural/unmanaged interference affects the cooperation process. We also consider maximum ratio combining (MRC) at the destination node and distributed space-time coding (DSTC) to mitigate the associated bandwidth overhead. Furthermore, to facilitate analysis, we just consider interference at the relays and ignore the effect of interference at the destination node, which matches the approach in [13]. Moreover, this study is targeted at legacy systems where $\max (\min (\cdot, \cdot))$ type policies are used for relay selection. Second, we focus upon two selection schemes to select two or four relays from a single group of relays. We derive new outage probability expressions for two or four relay selection and compare them with the results for conventional best single relay selection. Finally, we examine by simulation the bit error rate (BER) performance of the best single relay selection scheme and the best two-relay selection scheme, in the presence of errors in the feedback of relay selection information. In practice, this could be as simple as a single permission to transmit bit.

The remainder of this article is organized as follows. The system model and a statistical expression for interferencebased AF are described in Section 2. In Section 3, the relay selection criteria for interference-limited systems and asymptotic outage probability analysis are presented. Simulation results for outage probability analysis and impact of relay selection feedback errors are provided in Section 4. And conclusions are drawn in Section 5.

Notations: The following notations are used in the article. $\varepsilon(\cdot)$ represents the statistical expectation operator. A complex zero mean additive white Gaussian noise $n \sim$ $C N\left(0, N_{0}\right)$, where $N_{0}$ is the noise variance; and $\operatorname{Pr}(A)$ is the probability of $A . \Gamma(n)$ is the Gamma function, and $\mathbb{R}(\cdot)$ denotes the real value.

\section{System model}

Figure 1 shows two neighboring clusters of nodes denoted $(\mathrm{C} 1, \mathrm{C} 2)$ as in [13]. We focus on the analysis of the effect of inter-cluster interference on the relays in cluster $\mathrm{C} 1$ which

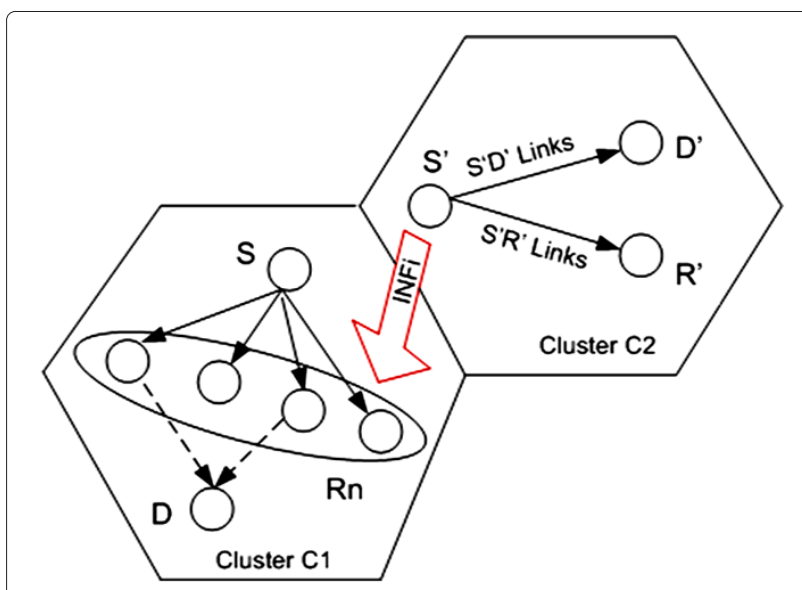

Figure 1 The system model. C1: cluster of interest, which contains a cooperative network which uses best two-relay selection. S: source; D: Destination; Rn: potential relay group. C2: neighboring cluster, S': source; $D^{\prime}$ : Destination. INFi: interference signal for the ith relay $\left(S^{\prime} \rightarrow \mathrm{Rn}\right)$.

contains nodes linked by independent Rayleigh flat-fading quasi-static channels. In this cluster, there is one source node and one destination node and many potential relay nodes grouped together, all equipped with single halfduplex antennas. Similar relay configurations have been studied in [16].

For simplicity of exposition, there is no direct link between the source and the destination as path loss or shadowing is assumed to render it unusable [17] and the neighboring cluster uses direct transmission from the source to a relay or the destination. In our protocol, the source broadcasts the signal to the relay nodes during the first time slot, and during the second time slot, the two (or four) selected relays, from the available group, transmit the received signal to the destination node. Moreover, the interference which is generated by the neighboring cluster is assumed only to affect the relay node and is ignored at the destination node. Therefore, the system model can be developed as follows: the received signal at the $i$ th relay and the destination node are given by

$$
\begin{aligned}
& y_{S R_{i}}=\sqrt{E_{S}} h_{S R_{i}} x+\sqrt{E_{S}} h_{S^{\prime} R_{i}} x^{\prime}+n_{R_{i}}, \\
& y_{R_{i} D}=\sqrt{P_{i}} h_{R_{i} D} y_{S R_{i}}+n_{D},
\end{aligned}
$$

where $x$ and $x^{\prime}$ are the source signals from the target and neighboring clusters, respectively, typically drawn from a prescribed finite constellation. $E_{S}$ is the average energy per symbol; $h_{S R_{i}}, h_{S^{\prime} R_{i}}$, and $h_{R_{i} D}$ are channel gains, which are the zero mean, independent, circularly symmetric, complex Gaussian random variables with variances $\sigma_{S R i}^{2}$, $\sigma_{S^{\prime} R_{i}}^{2}$, and $\sigma_{R_{i} D}^{2}$, between the source and the $i$ th relay node, between the neighboring source and the relay node and between the $i$ th relay node and the destination node; and 
the complex additive white Gaussian noise $n_{R_{i}}$ and $n_{D}$ are modeled as zero-mean mutually independent, circularlysymmetric, complex Gaussian random variables with variance $N_{0}$ at the $i$ th relay and the destination node, respectively. The $i$ th relay gain denoted by $\sqrt{P_{i}}$ is calculated from

$$
P_{i}=E_{S} /\left(E_{S}\left|h_{S R_{i}}\right|^{2}+E_{S}\left|h_{S^{\prime} R_{i}}\right|^{2}+N_{0}\right) .
$$

In our model, the source powers at the target and the neighboring cluster are assumed to be the same. This model is representative of an ad-hoc network environment where there is no power control between adjacent clusters.

Next, because the two- or four-relay selection scheme is used, we assume that MRC is used at the destination [18]. The practical implementation of the MRC will, however, incur a capacity penalty due to the need to adopt a time multiplexing approach to transmission between the relay and the destination nodes; however, this can be mitigated by adopting a DSTC [16], which is available for two or four relays. Furthermore, increasing the number of selected relays will incur practical overheads such as increased complexity in synchronization. Therefore, this article focuses on the selection of two and four relays. Therefore, the instantaneous equivalent end-toend signal-to-interference plus noise ratio (SINR) can be written as

$$
\gamma_{D}=\sum_{i \in N_{s}} \frac{P_{i}\left|h_{S R_{i}}\right|^{2}\left|h_{R_{i} D}\right|^{2}}{P_{i}\left|h_{S^{\prime} R_{i}}\right|^{2}\left|h_{R_{i} D}\right|^{2}+P_{i}\left|h_{R_{i} D}\right|^{2} N_{0}+N_{0}},
$$

where $N_{s}$ denotes the set of two or four relay indices for the relays chosen in our relay selection scheme, and each term in the summation has the same form as in [13]. Substituting (3) into (4), the end-to-end SINR is

$$
\gamma_{D}=\sum_{i \in N_{s}} \frac{\gamma_{S R_{i}} \gamma_{R_{i} D}}{\gamma_{I N F i}\left(\gamma_{R_{i} D}+1\right)+\gamma_{S R_{i}}+\gamma_{R_{i} D}+1},
$$

where $\gamma_{S R_{i}}=\left|h_{S R_{i}}\right|^{2} E_{S} / N_{0}$ and $\gamma_{R_{i} D}=\left|h_{R_{i} D}\right|^{2} E_{S} / N_{0}$ are the instantaneous SNRs of the source to $i$ th relay and $i$ th relay to destination links, respectively. And $\gamma_{I N F i}=$ $\gamma_{S^{\prime} R_{i}}=\left|h_{S^{\prime} R_{i}}\right|^{2} E_{S} / N_{0}$ denotes the interference-to-noise ratio (INR) for the $i$ th relay as a result of the neighboring source. It is difficult to use (5) to find a closed form expression for the probability density function (PDF) of $\gamma_{D}$, therefore, for high SNR, we provide an asymptotic bound as

$$
\gamma_{D} \simeq \sum_{i \in N_{s}} \frac{\gamma_{S R_{i}}}{\gamma_{I N F i}}
$$

which is the sum of the ratios between the SNR of the first hop and the INR of the interference, because when $\mathrm{SNR} \rightarrow \infty$, then $\varepsilon\left(\gamma_{I N F i}\right) \varepsilon\left(\gamma_{R_{i} D}\right) \gg \varepsilon\left(\gamma_{I N F i}\right)+\varepsilon\left(\gamma_{S R_{i}}\right)+$ $\varepsilon\left(\gamma_{R_{i} D}\right)+1$. In this case, the statistical description of the system is independent of the second hop.
For this asymptotic case, the PDF and cumulative distribution function (CDF) of each ratio in (6), which is between two exponential random variables [19], are given in closed form, as

$$
f(\gamma)=\frac{L}{(L+\gamma)^{2}} \quad \text { and } \quad F(\gamma)=\frac{\gamma}{(L+\gamma)},
$$

where $f(\cdot)$ and $F(\cdot)$ denote the PDF and the CDF, respectively. The parameter $L=\frac{\varepsilon\left(\gamma_{S R_{i}}\right)}{\varepsilon\left(\gamma_{I N F i}\right)}=\frac{\sigma_{S R_{i}}^{2}}{\sigma_{S^{\prime} R_{i}}^{2}}$. Note that the parameter $L$ controls the level of interference in the target and neighboring clusters.

Furthermore, considering interference at both the relays and the destination nodes is beyond the scope of this study and is left for analysis in future work. Our two- or fourrelay selection scheme assuming interference only at the relays will be implemented as presented in the following sections.

\section{Two- or four-relay selection with outage probability analysis}

In order to introduce our proposed two- or four-relay selection schemes, we need to introduce first the conventional relay selection scheme.

\subsection{Conventional relay selection}

In [11], the conventional relay selection policy which is used in the ideal distributed implementation without interference is considered. It requires the instantaneous signal SNR between the links from the source to relay and the relay to the destination node to be known, and then a particular relay is selected to maximize the minimum instantaneous SNR between them; the relay selection scheme can therefore be represented by

$$
b_{\text {con }}=\arg \max _{i \in N} \min \left(\gamma_{S R_{i}}, \gamma_{R_{i} D}\right),
$$

where $N$ represents the set of indices of all available relays.

The conventional relay selection policy offers the relay with the "best" end-to-end path between source and destination and provides diversity gain on the order of the number of the relays [12]. However, this relay selection criterion is only considered for environments without interference, and the best relay selection is not always sufficient to achieve the required outage probability at a destination node. Finally, when feedback error is present in the relay selection, the performance of the single relay selection scheme is significantly degraded, further discussion of which will be given in the simulation section. Therefore, to overcome these problems two- and fourrelay selection schemes are proposed for use in interference configurations for legacy networks which are restricted to adopt a $\max (\min (\cdot, \cdot))$ type policy. 


\subsection{Asymptotic two- and four-relay selection criterion}

The first proposed two- and four-relay selection criterion is motivated by the simplified expression of the system. As has been seen in (6), the asymptotic behavior of the system converges to the sum of the ratios between source to relay and interference links. Therefore, a relay selection policy is to choose the best relay set which gives the maximum value of the ratio. Take the best two relay selection for example, the asymptotic selection policy can be obtained as

$$
\mathbf{b}_{A s y}=\arg \max _{i \in N} \max _{i^{\prime} \in N-1}\left(\frac{\gamma_{S R_{i}}}{\gamma_{I N F i}}\right),
$$

where $\mathbf{b}=\left(i, i^{\prime}\right)$, i.e., the best pair of relay indices, where $i$ denotes the index of the relay with the best link in $N$, and $i^{\prime}$ is that of the best relay among the remaining $N-1$. In this approach, we select the best two relay nodes from the $N$ available relays in the group in the cluster, namely, select the relays with the maximum $\gamma_{\max }$ and the second largest $\gamma_{\max -1}$ from the $N$ relays instantaneous SNRs. Using the theory of order statistics [20], the selection of the maximum and the second largest is not independent, we therefore can find the joint distribution of the two largest values as

$$
f(x, y)=N(N-1) F(y)^{N-2} f(x) f(y),
$$

where we denote $\gamma_{\max }=x$ and $\gamma_{\max -1}=y$. Substituting (7) into (10), we obtain

$$
f(x, y)=\frac{N(N-1) L^{2} y^{N-2}}{(L+y)^{N}(L+x)^{2}} .
$$

Then we calculate the CDF $F_{\gamma_{\text {up }}}^{\text {Asy }}(\gamma)$, where $\gamma_{\text {up }}$ is the sum of $x$ and $y$, which are identically distributed and formed as the ratios of exponential random variables. Therefore, the CDF is obtained as

$$
F_{\gamma_{\text {up }}}^{\text {Asy }}(\gamma)=\operatorname{Pr}\{x+y \leq \gamma\} .
$$

Given that $x$ and $y$ are non-negative, with $x \geq y$, then,

$$
F_{\gamma_{\text {up }}}^{\text {Asy }}(\gamma)=\int_{0}^{\frac{\gamma}{2}} \int_{y}^{\gamma-y} f(x, y) d x d y \text {. }
$$

Using the PDF in (11), and after performing some manipulations, we obtain

$$
\begin{aligned}
F_{\gamma \text { up }}^{\text {Asy }}(\gamma)= & N(N-1) L^{2}\left\{\frac{\left(\frac{\gamma}{2}\right)^{N-1}\left(L N+\frac{\gamma}{2}\right)}{N(N-1) L^{2}\left(L+\frac{\gamma}{2}\right)^{N}}\right. \\
& -\frac{\left(\frac{\gamma}{2}\right)^{N}}{\left(L+\frac{\gamma}{2}\right)^{N}(L+\gamma)^{2}}\left[\frac{2(L+\gamma)\left(L+\frac{\gamma}{2}\right)}{L(N-1) \gamma}\right. \\
& +\frac{\left(\frac{L+\frac{\gamma}{2}}{L}\right)^{N} F_{2,1}\left(N, N ; N+1 ;-\frac{\gamma}{2 L}\right)}{N} \\
& \left.\left.+\frac{\frac{\gamma}{2}\left(\frac{L+\frac{\gamma}{2}}{L}\right)^{N} F_{1}\left(N+1 ; N, 1 ; N+2 ;-\frac{\gamma}{2 L}, \frac{\gamma}{2(L+\gamma)}\right)}{L N+L+\gamma N+\gamma}\right]\right\},
\end{aligned}
$$

where $F_{2,1}(a, b, c, z)$ is the first hypergeometric function, which can be calculated by using the Hypergeom Matlab function [21]. Furthermore, $F_{1}(a ; b 1, b 2 ; c ; x, y)$ is a formal extension of the Appell hypergeometric function of two variables, which can also be expressed by the simple integral in [22] as

$$
\begin{aligned}
F_{1}(a ; b 1, b 2 ; c ; x, y)= & \frac{\Gamma(c)}{\Gamma(a) \Gamma(c-a)} \\
& \times \int_{0}^{1} t^{a-1}(1-t)^{c-a-1}(1-x t)^{-b_{1}}(1-y t)^{-b_{2}} d t \\
& \quad \text { for } \mathbb{R}(c)>\mathbb{R}(a)>0,
\end{aligned}
$$

where $\Gamma(n)=(n-1)$ ! is the Gamma function.

Therefore, the outage probability is defined as when the average end-to-end SNR falls below a certain predefined threshold value, $\alpha$. The outage probability can be expressed as

$$
P_{\text {out }}=\int_{0}^{\alpha} f_{\gamma}(\gamma) d \gamma=F_{\gamma_{u p}}(\alpha)
$$

The outage probability of the best two-relay selection can be expressed by using the CDF expression (14).

Then we can use a similar method to obtain the outage probability for the best four-relay selection as follows. The asymptotic selection policy can be obtained as

$$
\mathbf{b}_{\mathrm{con}}=\arg \max _{i \in N} \max _{i^{\prime} \in N-1} \max _{i^{\prime \prime} \in N-2} \max _{i^{\prime \prime \prime} \in N-3}\left(\frac{\gamma_{S R_{i}}}{\gamma_{I N F i}}\right),
$$

where $\mathbf{b}=\left(i, i^{\prime}, i^{\prime \prime}, i^{\prime \prime \prime}\right)$, i.e., the best four relay indices, in which $i$ denotes the index of the relay with the best link in $N ; i^{\prime}$ is that of the best relay among the remaining $N-1$, and $i^{\prime \prime}$ is that of the best relay among the remaining $N-2$, and $i^{\prime \prime \prime}$ is that of the best relay among the remaining $N-3$. The joint distribution of the four largest values is

$f(w, x, y, z)=N(N-1)(N-2)(N-3) F(z)^{N-4} f(w) f(x) f(y) f(z)$,

where we define $\gamma_{\max }=w, \gamma_{\max -1}=x, \gamma_{\max -2}=y$ and $\gamma_{\max -3}=z$. Substituting (7) into (17), we can obtain

$$
f(w, x, y, z)=\frac{N(N-1)(N-2)(N-3) L^{4} z^{N-4}}{(L+z)^{N-2}(L+w)^{2}(L+x)^{2}(L+y)^{2}} .
$$

Then we calculate the CDF $F_{\gamma_{\text {up }}}^{\text {Asy }}(\gamma), \gamma_{\text {up }}$ is formed as the sum of $w, x, y$, and $z$ random variables, which are identically distributed and ratios of exponential random variables. Therefore, the CDF is obtained by

$$
F_{\gamma_{\text {up }}}^{\text {Asy }}(\gamma)=\operatorname{Pr}\{w+x+y+z \leq \gamma\} .
$$


Given that $w, x, y$, and $z$ are non-negative, with $w \geq x \geq$ $y \geq z$, then,

$$
\begin{aligned}
F_{\gamma_{\text {up }}}^{\text {Asy }}(\gamma)= & \int_{0}^{\frac{\gamma}{4}} \int_{z}^{\frac{\gamma-z}{3}} \int_{y}^{\frac{\gamma-z-y}{2}} \\
& \times \int_{x}^{\gamma-z-y-x} f(w, x, y, z) d w d x d y d z .
\end{aligned}
$$

Substituting (18) into (20), we can obtain

$$
\begin{aligned}
F_{\gamma_{\text {up }}}^{\text {Asy }}(\gamma)= & \int_{0}^{\frac{\gamma}{4}} \int_{z}^{\frac{\gamma-z}{3}} \int_{y}^{\frac{\gamma-z-y}{2}} \int_{x}^{\gamma-z-y-x} \\
& \times \frac{N(N-1)(N-2)(N-3) L^{4} z^{N-4}}{(L+z)^{N-2}(L+w)^{2}(L+x)^{2}(L+y)^{2}} d w d x d y d z .
\end{aligned}
$$

Then, exploiting (21) as in (15), the outage probability can be evaluated, for example for the results in Section 4 we employ the Mathematica software package [23].

In this study, we focus on a two- or four-relay selection approach as it is immediately applicable within a cooperative network, which exploits DSTC [16] to improve the end-to-end performance, such as an Alamouti or QuasiOrthogonal code, according to the number of selected relays. Furthermore, for our relay selection policy, it requires only the SNR of the links from source to relay nodes and the INR of the interference links which can be obtained by the relay nodes during the early stage of transmission. In terms of the relay selection policy, moreover, the information describing the links between the relay and destination is not required at the destination node, therefore, this policy has a lower complexity than that of [12] and may save feedback set-up time.

\subsection{Semi-conventional two- and four-relay selection}

The semi-conventional two- and four-relay selection schemes are an extension of the conventional selection scheme and motivated by the expression of the general statistics (4). There are three advantages in the semi-conventional two- and four-relay selection scheme. First, because this scheme is based on the conventional approach, it does not involve complex computational operations, and can easily be obtained from the conventional case without modifying the $\min (\cdot, \cdot)$ operation. Second, it is suitable for ad-hoc systems with mobility that dynamically and continuously change between interference and non-interference environments. Third, the proposed scheme balances the gap between the conventional scheme and asymptotic case for the interference situation. Therefore, in this study, we just focus on a simple ratio between the conventional $\min (\cdot, \cdot)$ operation and the interference term, because it does not change the basic structural core of the system. In the following, the best two-relay selection scheme becomes

$$
\mathbf{b}_{\text {Semi }}=\arg \max _{i \in N} \max _{i^{\prime} \in N-1}\left(\frac{\min \left(\gamma_{S R_{i}}, \gamma_{R_{i} D}\right)}{\gamma_{I N F i}}\right)
$$

where $\mathbf{b}=\left(i, i^{\prime}\right)$, i.e., the best pair of relay indices, where $i$ denotes the index of the relay with the best link in $N$, and $i^{\prime}$ is that of the best relay among the remaining $N-1$. Here, we need to consider the outage behavior of the ratio $\frac{\gamma_{S R_{i}}}{\gamma_{I N F i}}$ according to the semi-conventional scheme. In order to simplify the approximation of the corresponding outage bound as in [13], two cases will be considered.

In the first case, the value $\min \left(\gamma_{S R_{i}}, \gamma_{R_{i} D}\right)=\gamma_{S R_{i}}$, which means the selected relay, the minimum between the two hops, is the link between source and relay. Therefore, the PDF and CDF are given in closed form and correspond to a ratio between the min operation and an exponential random variable, which are

$$
f(\gamma)=\frac{2 L}{(L+2 \gamma)^{2}} \text { and } F(\gamma)=\frac{2 \gamma}{L+2 \gamma},
$$

where $f(\cdot)$ and $F(\cdot)$ denote the PDF and the CDF, respectively. Substituting (23) into (10), we obtain

$$
f(x, y)=\frac{N(N-1) 2^{N} L^{2} y^{N-2}}{(L+2 y)^{N}(L+2 x)^{2}} .
$$

Using the PDF in (24) and (13), and after performing some manipulations, we can obtain (25), some definitions for which have already been shown in the last section.

$$
\begin{aligned}
F_{\gamma_{\text {up }}}^{\prime}(\gamma)= & N(N-1) 2^{N} L^{2}\left\{\frac{\left(\frac{\gamma}{2}\right)^{N-1}(L N+\gamma)}{2 N(N-1) L^{2}(L+\gamma)^{N}}\right. \\
& -\frac{\left(\frac{\gamma}{2}\right)^{N}}{2(L+\gamma)^{N}(L+2 \gamma)^{2}}\left[\frac{2(L+2 \gamma)(L+\gamma)}{L(N-1) \gamma}\right. \\
& +\frac{2\left(\frac{L+\gamma}{L}\right)^{N} F_{2,1}\left(N, N ; N+1 ;-\frac{\gamma}{L}\right)}{N} \\
& \left.\left.+\frac{2 \gamma\left(\frac{L+\gamma}{L}\right)^{N} F_{1}\left(N+1 ; N, 1 ; N+2 ;-\frac{\gamma}{L}, \frac{\gamma}{L+2 \gamma}\right)}{(N+1)(L+2 \gamma)}\right]\right\} .
\end{aligned}
$$

In the second case, the value $\min \left(\gamma_{S R_{i}}, \gamma_{R_{i} D}\right)=\gamma_{R_{i} D}$, which means the minimum between the two hops of the selected relay, is the link from relay to destination which is not considered in the ratio of interest. According to the assumption of [13], the conventional asymptotic relay selection can be used as an outage bound in this case. Therefore, based on the above two equiprobable cases, the semi-conventional end-to-end CDF is given as

$$
F_{\gamma_{\text {up }}}^{\text {Semi }}(\gamma)=\frac{1}{2} F_{\gamma_{\text {up }}}^{\text {Asy }}(\gamma)+\frac{1}{2} F_{\gamma_{u p}}^{\prime}(\gamma)
$$

where $F_{\gamma_{\text {up }}}^{\text {Asy }}(\gamma)$ and $F_{\gamma_{\text {up }}}^{\prime}(\gamma)$ are denoted by (14) and (25), respectively. And the outage probability can be obtained by using (26). 
Then, we can use a similar method to obtain the best four relays in the following processing. The semiconventional selection policy can be obtained as

$\mathbf{b}_{\text {Semi }}=\arg \max _{i \in N} \max _{i^{\prime} \in N-1} \max _{i^{\prime \prime} \in N-2} \max _{i^{\prime \prime \prime} \in N-3}\left(\frac{\min \left(\gamma_{S R_{i}}, \gamma_{R_{i} D}\right)}{\gamma_{I N F i}}\right)$,

where $\mathbf{b}=\left(i, i^{\prime}, i^{\prime \prime}, i^{\prime \prime \prime}\right)$, i.e., the best four relay indices, wherein $i$ denotes the index of the relay with the best link in $N ; i^{\prime}$ is that of the best relay among the remaining $N-1$, and $i^{\prime \prime}$ is that of the best relay among the remaining $N-2$, and $i^{\prime \prime \prime}$ is that of the best relay among the remaining $N-3$. In the first case, the joint distribution of the four largest values can be obtained by substituting (23) into (17), yielding

$f(w, x, y, z)=\frac{N(N-1)(N-2)(N-3) 2^{N} L^{4} z^{N-4}}{(L+2 z)^{N-2}(L+2 w)^{2}(L+2 x)^{2}(L+2 y)^{2}}$.

Substituting (28) into (20), and after performing some manipulations, we can obtain

$$
\begin{aligned}
F_{\gamma_{\text {up }}}^{\prime}(\gamma)= & \int_{0}^{\frac{\gamma}{4}} \int_{z}^{\frac{\gamma-z}{3}} \int_{y}^{\frac{\gamma-z-y}{2}} \int_{x}^{\gamma-z-y-x} \\
& \times \frac{N(N-1)(N-2)(N-3) 2^{N} L^{4} z^{N-4}}{(L+2 z)^{N-2}(L+2 w)^{2}(L+2 x)^{2}(L+2 y)^{2}} d w d x d y d z .
\end{aligned}
$$

Therefore, the final end-to-end CDF can be obtained as

$$
F_{\gamma_{\mathrm{up}}}^{\mathrm{Semi}}(\gamma)=\frac{1}{2} F_{\gamma_{\mathrm{up}}}^{\mathrm{Asy}}(\gamma)+\frac{1}{2} F_{\gamma_{u p}}^{\prime}(\gamma)
$$

where $F_{\gamma_{\text {up }}}^{\text {Asy }}(\gamma)$ and $F_{\gamma_{\text {up }}}^{\prime}(\gamma)$ are given by (21) and (29), respectively. And the outage probability can be evaluated by using (15) and (30), for example with the Mathematica software package [23].

\section{Simulation results for outage probability analysis and impact of relay selection feedback errors}

In this section, in order to verify the results obtained from the above mathematical expressions, we assumed the target source node and the neighboring source node use the same unity transmission power, and there is no direct link between the source and the destination as path loss or shadowing render it unusable. We show outage probability performance of the two- and four-relay selection schemes.

Figure 2 shows the comparison of the outage probability of the best two-relay selection schemes, where $L=$ 5 and 20. It can be seen that increasing the number of relays, $N$, decreases the outage probability, and hence when the number of relays is large, the outage event (no transmission) becomes less likely, for example, with the total number of available relays increasing from 4 to 6 ,

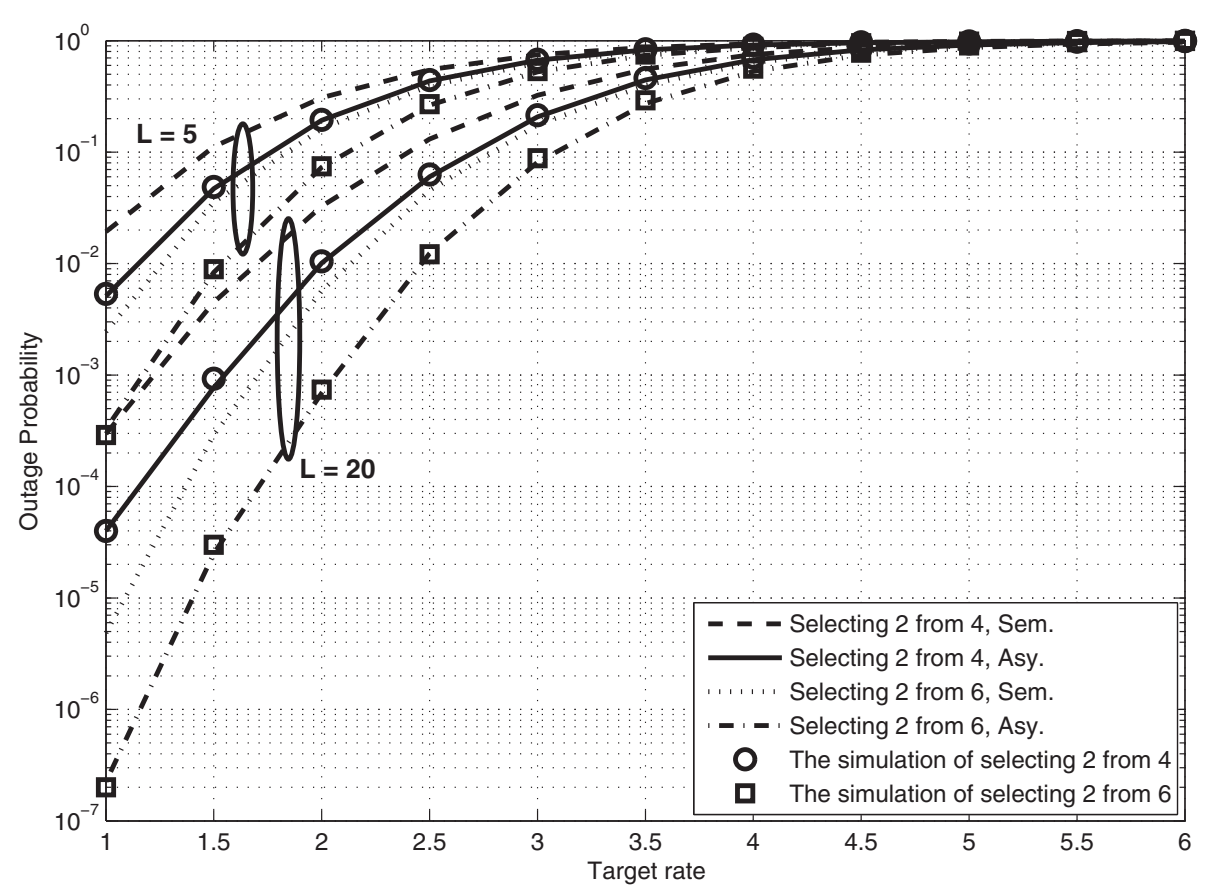

Figure 2 Comparison of the outage probability of the best two-relay selection schemes, the theoretical results are shown in line style and the simulation results as points. 
the outage probability of the best two-relay selection is decreased from approximately 0.308 to 0.162 for the semi-conventional case; and from 0.192 to 0.073 for the asymptotic case when the threshold value $\alpha$ is $15 \mathrm{~dB}$ and $L=5$. The outage performance of the asymptotic case closely matches the simulation results, when $\mathrm{SNR}=40 \mathrm{~dB}$. Moreover, with increased source-to-interference power ratio, the performance in terms of outage probability is improved.

Figure 3 shows the outage probability of the best fourrelay selection schemes, where $L=5$ and 20. It can be seen that increasing the number of relays, decreases the outage probability, for example, with the total number of available relays increasing from 6 to 8 , the outage probability of the best four-relay selection is decreased from approximately 0.013 to 0.0025 for the semi-conventional case; and from 0.0022 to $1.6 \times 10^{-4}$ for the asymptotic case when the threshold value $\alpha$ is $7 \mathrm{~dB}$ and $L=5$. With increased source-to-interference power ratio, the performance of outage probability again improves. Moreover, the asymptotic results match very well with the simulation results, when $\mathrm{SNR}=40 \mathrm{~dB}$.

Figure 4 shows the comparison of the outage probability of the single relay selection and the best two- and four-relay selection schemes, $\mathrm{SNR}=40 \mathrm{~dB}$ and $L=5$ or 10. Obviously, with increasing the number of selected relays, the outage probability decreases. For example, for the semi-conventional case, when the total number of available relays is $6, L=5$ and the threshold value $\alpha$ is $7 \mathrm{~dB}$, the outage probability of a single relay, the best two-relay and the best four-relay selections are approximately $0.1,0.036$, and 0.013 , respectively. Furthermore, for the asymptotic case, when $N=6, L=10$, and $\alpha=7 \mathrm{~dB}$, the outage probability of the best relay, the best two-relay, and the best four-relay selections are approximately $0.0045,5.59 \times 10^{-4}$, and $9.01 \times 10^{-5}$, respectively. These results confirm that two- and four-relay selection schemes provide more robust transmission than single relay selection, because for the single relay selection, it just uses a single relay to help the source to transmit the signal. Therefore, we can choose a different number of relays to communicate with the source and destination nodes, according to the target outage probability. Finally, through using DSTC we have the advantage of avoiding the bandwidth overhead in using the MRC scheme. We next consider how the end-to-end BER performance of the relay selection schemes degrades when there is an error in selecting the particular relay(s) to use in transmission.

Next, we compare the BER performance of the best tworelay selection from a group of $N$ available relays, $N=$ 4, with distributed Alamouti code with the best single relay selection in the presence of relay selection feedback errors, when quadrature phase-shift keying symbols are used in transmission.

We want to show the comparison between the best two-relay selection and the single relay selection in a

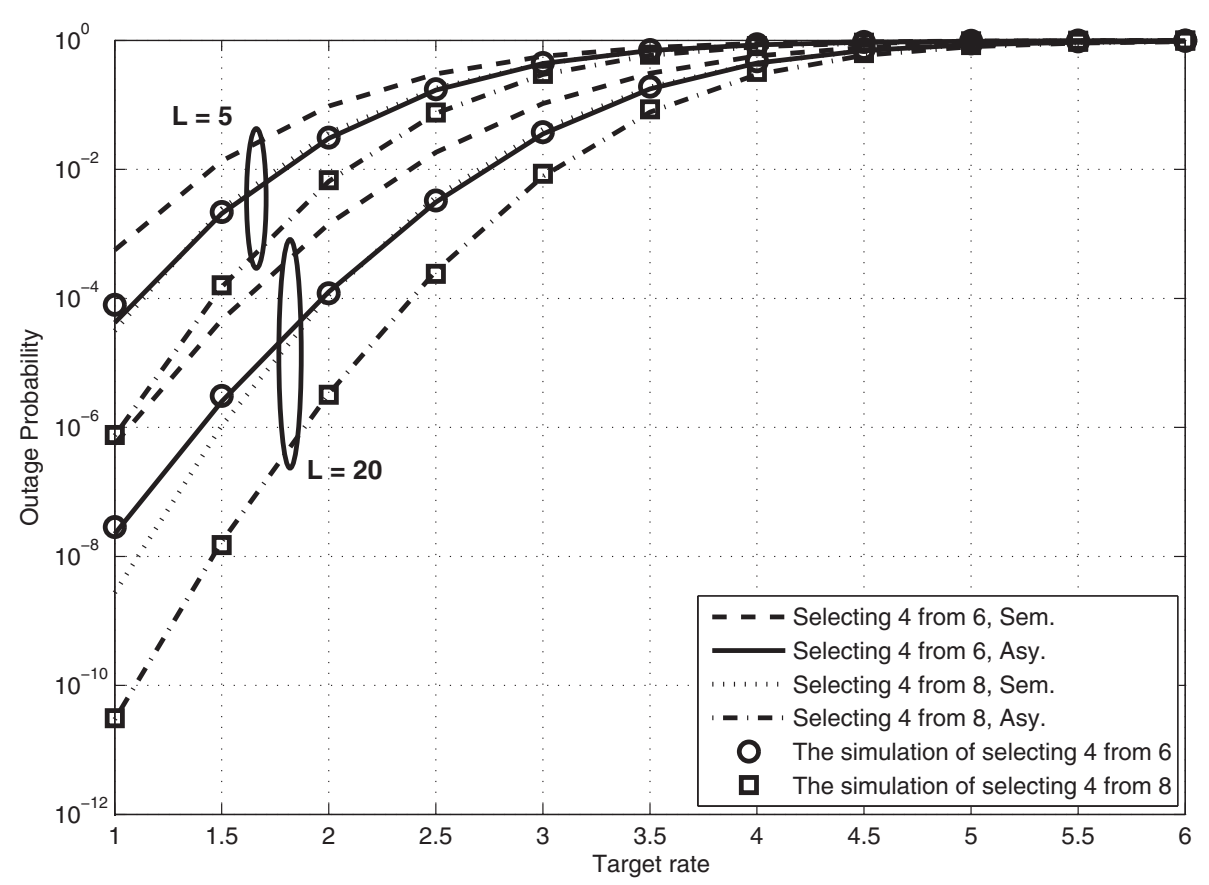

Figure 3 Comparison of the outage probability of the best four-relay selection schemes, the theoretical results are shown in line style and the simulation results as points. 


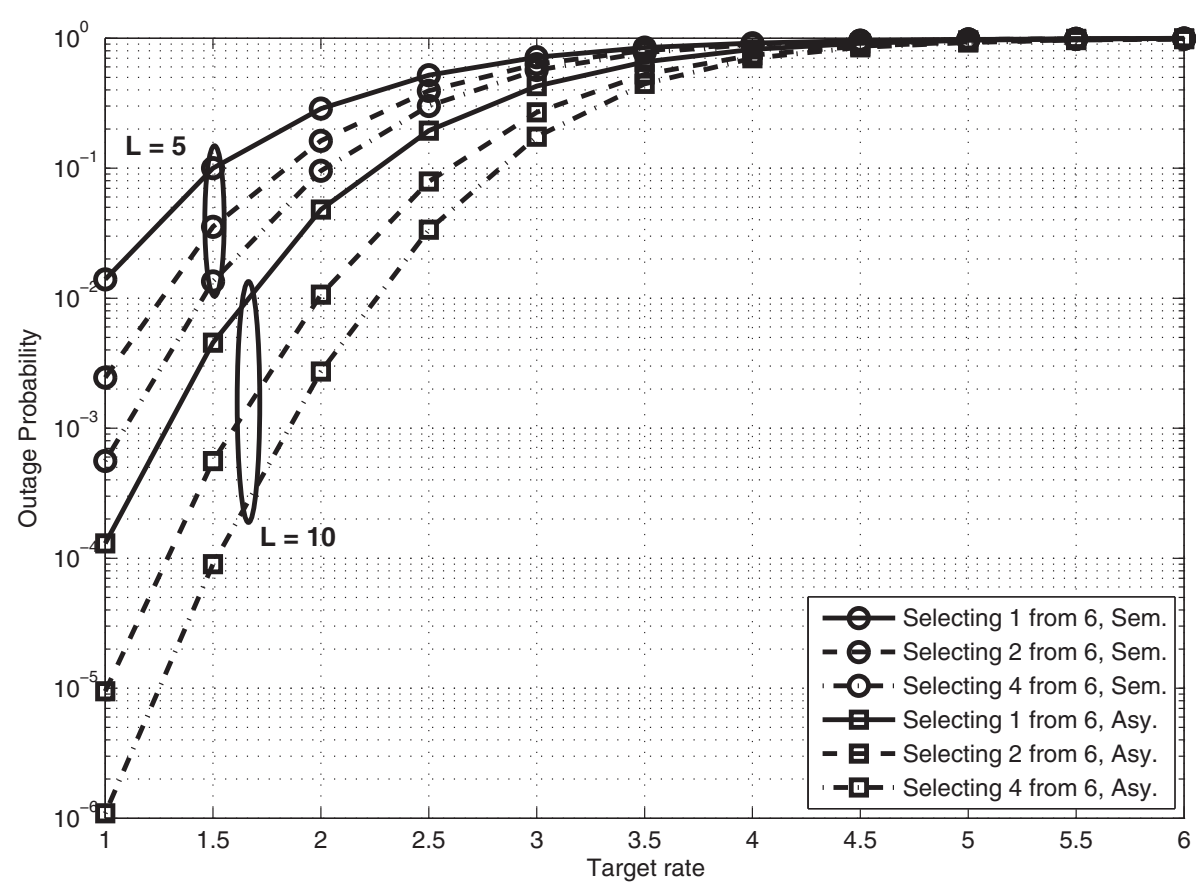

Figure 4 Comparison of the outage probability of the single relay selection and the best two- and four-relay selection schemes.

representative relay selection feedback error environment, and the signal-to-interference power ratio $L=50$ is assumed. To simulate errors in the feedback of relay selection information from the destination we introduce an error rate in the feedback. An error rate of 0.5 corresponds to $50 \%$ of the selections being made in error; that is, rather than selecting the best relay, one of the other relays is chosen with equal probability of selection. As can be seen in Figure 5, when perfect relay selection is made, i.e., an error rate of 0 , the BER performance of the best single relay

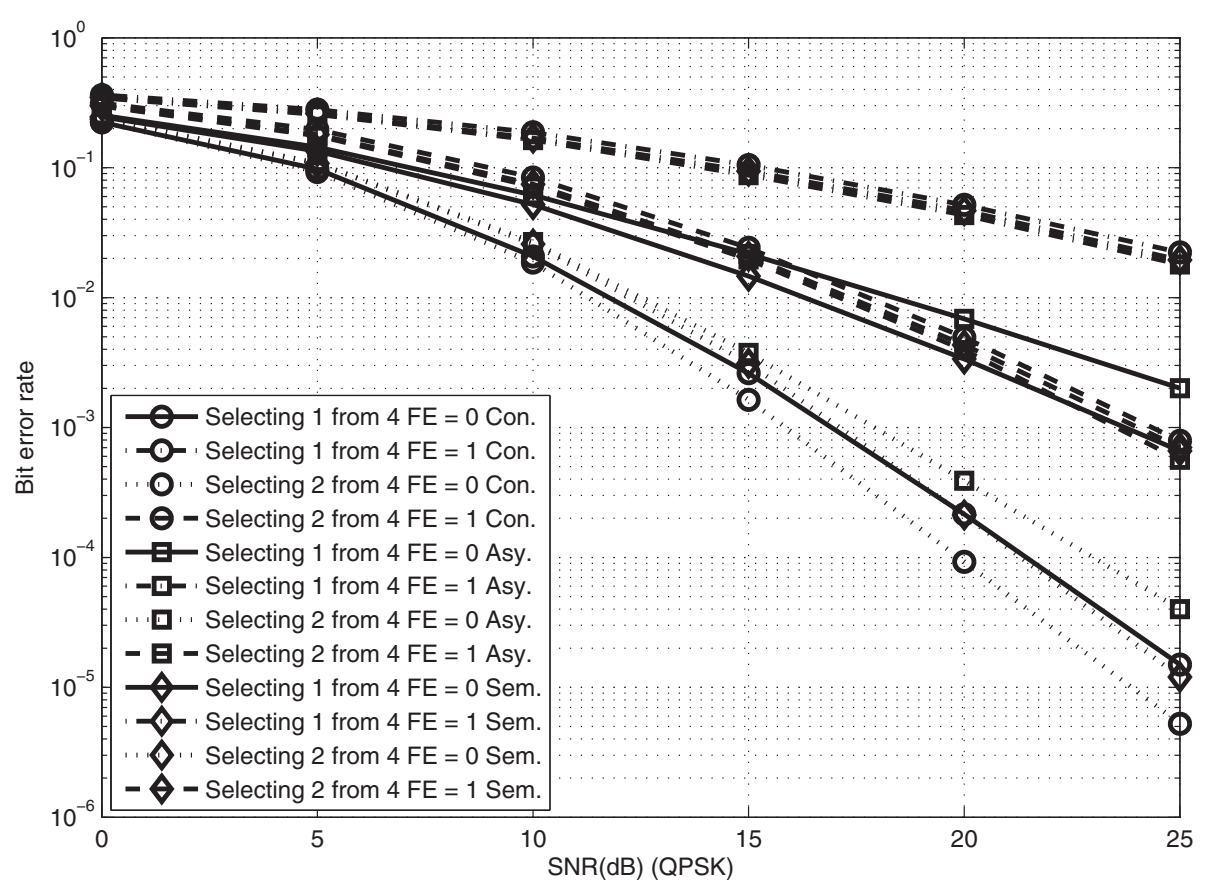

Figure 5 BER performance comparison of different best two-relay selection schemes with the different best single relay selection schemes, with varying error in the feedback relay selection information from the destination, where $L=\mathbf{5 0}$. 
selection is worse than the best two-relay selection for the three different relay selection schemes, which are denoted by circular, square, and diamond dotted lines for the conventional, asymptotic, and semi-conventional schemes, respectively. Moreover, in the presence of errors in the relay selection, i.e., error rate over the range 0 to 1 , all of the different best two-relay selection schemes outperform that of the best single relay selection. These results illustrate clearly the increased robustness of the best two-relay selection scheme over the single relay selection scheme in the presence of moderate to severe relay selection feedback errors. For example, for the conventional best tworelay selection scheme, when the SNR is $20 \mathrm{~dB}$, the BER for the conventional best two-relay selection changes from approximately $1 \times 10^{-4}$ only to $4.9 \times 10^{-3}$ as the error rate changes from 0 to 1 , whereas the BER for the single relay selection is increased from approximately $2.15 \times 10^{-4}$ to $5.1 \times 10^{-2}$, confirming the improved robustness.

Compared with Figure 5, Figure 6 has the same trend for BER performance, which confirms the increased robustness of the best two-relay selection scheme over the single relay selection scheme in the presence of moderate to severe relay selection feedback errors. For example, for the conventional best two-relay selection scheme, when the SNR is $20 \mathrm{~dB}$, the BER for the conventional best two-relay selection changes from approximately $2.4 \times 10^{-4}$ only to $8 \times 10^{-3}$ as the feedback error rate changes from 0 to 1 , whereas the BER for the single relay selection is increased from approximately $3.5 \times 10^{-4}$ to $6 \times 10^{-2}$, confirming the improved robustness. Moreover, because of the increased interference from the neighboring cluster, the BER performance of the three selection schemes is slightly worse than that of the small interference environment given in Figure 5, when $L=50$. For example, for the conventional, asymptotic, and semi-conventional best two-relay selection schemes, when $\mathrm{SNR}=25 \mathrm{~dB}$, the BER for the conventional, asymptotic, and semi-conventional best two-relay selection increase from approximately $5 \times 10^{-6}$ to $2 \times 10^{-5}$, from approximately $3.8 \times 10^{-5}$ to $4.2 \times 10^{-5}$, and from approximately $1.5 \times 10^{-5}$ to $3 \times 10^{-5}$ as the $L$ decreases from 50 to 10 .

\section{Conclusion}

We have examined two different selection schemes which are asymptotical and semi-conventional policies to select the best two and four relays from a group of available relays in the same cluster by using local measurements of the instantaneous channel conditions in the context of legacy systems which adopt $\max (\min (\cdot, \cdot))$ type policies. New analytical expressions for the PDF, and CDF of end-to-end SNR were derived together with closed form expressions for outage probability over Rayleigh fading channels. Numerical results were provided to show the advantage of the outage probability performance of the best two- and four-relay selection in a cooperative communication system. Moreover, through simulation study,

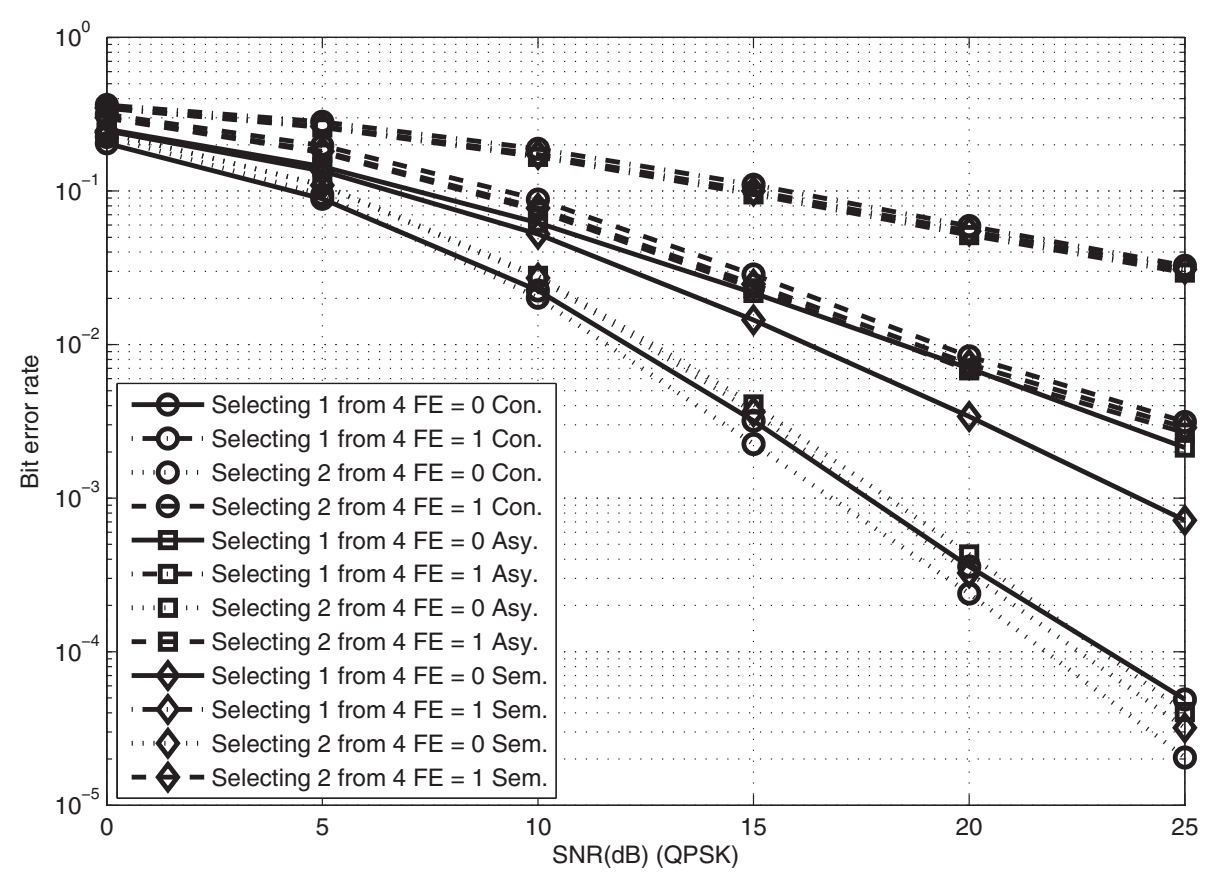

Figure 6 BER performance comparison of different best two-relay selection schemes with the different best single relay selection schemes, with varying error in the feedback relay selection information from the destination, where $L=10$. 
we confirmed the robustness of the best two-relay selection scheme in the presence of moderate to severe relay selection feedback errors.

\section{Competing interest}

The authors declare that they have no competing interests.

\section{Acknowledgements}

The authors would like to thank the anonymous reviewers and the editor for improving the clarity of this article.

Received: 7 June 2012 Accepted: 1 November 2012

Published: 21 November 2012

\section{References}

1. JN Laneman, GW Wornell, Distributed space-time-coded protocols for exploiting cooperative diversity in wireless networks. IEEE Trans. Inf. Theory. 49(10), 2415-2425 (2003)

2. M Dohler, YH Li, Cooperative Communications: Hardware, Channel and PHY (Wiley \& Sons Ltd., New York, 2010)

3. RU Nabar, H Bolcskei, FW Kneubuhler, Fading relay channels: performance limits and space-time signal design. IEEE J. Sel. Areas Commun. 22(6), 1099-1109 (2004)

4. AH Madsen, J Zhang, Capacity bounds and power allocation for wireless relay channels. IEEE Trans. Inf. Theory. 51, 2020-2040 (2005)

5. L Lai, K Liu, HE Gamal, The three-node wireless network: achievable rates and cooperation strategies. IEEE Trans. Inf. Theory. 52, 805-828 (2006)

6. SS Ikki, S Aissa, Multihop wireless relaying systems in the presence of cochannel interferences: performance analysis and design optimization. IEEE Trans. Veh. Technol. 61(2), 566-573 (2012)

7. S Ikki, S Aissa, Impact of imperfect channel estimation and co-channel interference on dual-hop relaying systems. IEEE Commun. Lett. 16(3), 324-327 (2012)

8. HA Suraweera, HK Garg, A Nallanathan, Performance analysis of two hop amplify-and-forward systems with interference at the relay. IEEE Commun. Lett. 14(8), 692-694 (2010)

9. Z Bali, W Ajib, H Boujemaa, in Proceedings of the IEEE ICT. Distributed relay selection strategy based on source-relay channel, Montreal, Canada, April 2010)

10. A Adinoyi, YJ Fan, H Yanikomeroglu, HV Poor, F Al-Shaalan, Performance of selection relaying and cooperative diversity. IEEE Trans. Wirel. Commun. 12(8), 5790-5795 (2009)

11. A Bletsas, A Knisti, DP Reed, A Lippman, A simple cooperative diversity method based on network path selection. IEEE J. Sel. Areas Commun. 24(3), 659-672 (2006)

12. A Bletsas, H Shin, MZ Win, Cooperative communications with outage-optimal opportunistic relaying. IEEE Trans. Wirel. Commun. 6(9), 3450-3460 (2007)

13. I Krikidis, JS Thompson, S McLaughlin, N Goertz, Max-min relay selection for legacy amplify-and-forward systems with interference. IEEE Trans. Wirel. Commun. 8(6), 3016-3027 (2009)

14. L Musavian, S Aissa, S Lambotharan, Effective capacity for interference and delay constrained cognitive radio relay channels. IEEE Trans. Wirel. Commun. 9(10), 1698-1707 (2010)

15. JN Laneman, D Tse, GW Wornell, Cooperative diversity in wireless networks: efficient protocols and outage behavior. IEEE Trans. Inf. Theory. 50(12), 3063-3080 (2004)

16. Y Jing, $\mathrm{H}$ Jafarkhani, Using orthogonal and quasi-orthogonal designs in wireless relay networks. IEEE Trans. Inf. Theory. 53(11), 4106-4118 (2007)

17. DS Michalopoulos, GK Karagianidis, Performance analysis of single relay selection in Rayleigh fading. IEEE Trans. Wirel. Commun. 7(11), 3718-3724 (2008)

18. PA Anghel, M Kaveh, Exact symbol error probability of a cooperative network in a Rayleigh-fading environment. IEEE Trans. Wirel. Commun. 3(5), 1416-1421 (2004)

19. S Nadarajah, S Kotz, On the product and ratio of Gamma and Weibull random variables. Econ. Theory (Cambridge University Press). 22, 338-344 (2006)

20. N Balakrishnan, AC Cohen, Order Statistics and Inference: Estimation Methods (Academic Press, London, 1991)
21. A Gilat, V Subramaniam, Numerical Methods for Engineers and Scientists: An Introduction with Applications Using MATLAB (Wiley \& Sons Ltd., New York, 2011)

22. WN Bailey, On the reducibility of Appell's function. Quart. J. Math. (Oxford). 5(1), 291-292 (1934)

23. S Wolfram, The Mathematica Book, 5th edn. (Wolfram Media Press, Champaign, Illinois, U.S.A., 2003)

doi:10.1186/1687-1499-2012-351

Cite this article as: Chen et al:: Two- and four-relay selection schemes for application in interference limited legacy networks. EURASIP Journal on Wireless Communications and Networking 2012 2012:351.

\section{Submit your manuscript to a SpringerOpen ${ }^{\circ}$ journal and benefit from:}

- Convenient online submission

- Rigorous peer review

- Immediate publication on acceptance

- Open access: articles freely available online

- High visibility within the field

- Retaining the copyright to your article

Submit your next manuscript at $\gg$ springeropen.com 